\title{
Anatomy of the Gall-Bladder in the Ultrasound about 1000 Cases
}

\author{
Bréhima Coulibaly1,2, Abdoulaye Kanté1,2, Abdoulaye Koné3, Bréhima Bengaly¹, Babou Ba², \\ Youlouza Coulibaly ${ }^{3}$, Oncoumba Diarra ${ }^{3}$, Adama Seriba Diarra1, Drissa Traoré1, \\ Mariam Daou ${ }^{4}$, Nouhoum Ongoïba ${ }^{1,2^{*}}$
}

\author{
${ }^{1}$ Service of Surgery B. CHU Point G, Bamako, Mali \\ ${ }^{2}$ Laboratory of Anatomy of the Faculty of Medicine and Odontostomatology, Bamako, Mali \\ ${ }^{3}$ Imaging Department CHU Point G, Bamako, Mali \\ ${ }^{4}$ Service of Neurology.CHU Gabriel Touré, Bamako, Mali \\ Email: kanteim@yahoo.fr, *ongoibano@yahoo.fr
}

How to cite this paper: Coulibaly, B., Kanté, A., Koné, A., Bengaly, B., Ba, B., Coulibaly, Y., Diarra, O., Diarra, A.S., Traoré, D., Daou, M. and Ongoïba, N. (2019) Anatomy of the Gall-Bladder in the Ultrasound about 1000 Cases. Forensic Medicine and Anatomy Research, 7, 44-49. https://doi.org/10.4236/fmar.2019.72008

Received: October 20, 2018

Accepted: December 23, 2018

Published: January 25, 2019

Copyright $\odot 2019$ by author(s) and Scientific Research Publishing Inc. This work is licensed under the Creative Commons Attribution International License (CC BY 4.0).

http://creativecommons.org/licenses/by/4.0/

\begin{abstract}
Aim: The aim of this work is to determine the dimensions and the capacity of the gall-bladder by ultrasound. Methodology: We realized a descriptive study over 2 years (from January, 2015 till December, 2017). It concerned an ultrasound exploration of the gall-bladder. The studied population was healthy voluntary subjects visible and on an empty stomach for 12 hours, without history of abdominal trauma and abdominal operating scar. The carrying subjects of the tracks of abdominal trauma or of abdominal operating scar were not included. Results: The average age was of 39 years \pm 12.16 with extreme of 10 years and 89 years. It was 500 women (50\%) and of 500 men $(50 \%)$ with a sex ratio of 1 . To 450 subjects (45\%) the width of the gall-bladder of the subjects varied between 21 and 30. The average was $22.63 \mathrm{~mm} \pm$ 7.9. Extremes were from 9 to $55 \mathrm{~mm}$. The measurements made by the three sonographers showed no significant difference. The sensitivity of ultrasound in the visualization of the gallbladder was $100 \%$. The length of the gall-bladder was between 61 and 70 $\mathrm{mm}$. The average was $65.35 \pm 14.48$. Extremes were 26 and $142 \mathrm{~mm}$. Conclusion: This original study shows the normal dimensions of the gall-bladder to Malian.
\end{abstract}

\section{Keywords}

Anatomy, Gall-Bladder, Ultrasound, Bamako-Mali

\section{Introduction}

The gall-bladder is a reservoir connected in diversion on the main biliary way. It 
is placed in the lower face of the liver in the cystic dimple. The ultrasound stays in private hospital its essential way of exploration. Few anatomical works were dedicated to it. The interest of this study is that the gall-bladder is an organ which is very requested in private hospital: it is an organ which often becomes infected and the led modifications by this infection are appreciated by the ultrasound where from the interest to know well the normal dimensions.

These measures are not made in the same conditions and they are variously estimated by the authors [1] [2] [3]. The anatomical works, in particular the ultrasound anatomy of the gall-bladder, centered on the dimensions (length, width and thickness of the wall), can help the African surgeons in the activities of surgery and coelio-surgery on the gall-bladder.

The purpose of this study was to determine the dimensions and the capacity of the gall-bladder by ultrasound.

\section{Materials and Methods}

We realized a descriptive study over 2 years (from January, 2015 till December, 2017). It concerned an ultrasound exploration of the gall-bladder. The studied population was healthy voluntary subjects visible and on an empty stomach for 12 hours, without history of abdominal trauma and abdominal operating scar. The carrying subjects of the tracks of abdominal trauma or of abdominal operating scar were not included. Subjects with morphological abnormalities were also excluded.

Three different sonographers made at the same time this examination by using the same protocol and type Aloka's same device SSD-1700 provided with a convex probe of 3.5 megahertzs and with a linear probe of 7.5 megahertzs. The subjects were installed in dorsal decubitus, the lengthened arms. The various probes are successively placed on the abdominal wall particularly at the level of the region of the right hypochondria. The liver was identified and its limits were marked by the cursor of the echograph. The probe was directed then to the lower edge of the liver for the location of the gall-bladder.

The gall-bladder was spotted and its dimensions (length, width, thickness of the wall) were measured. The dimensions were measured on a longitudinal incidence and they were expressed in millimeter. The capacity vesicular was obtained by marking all the limits of the gall-bladder by the cursor of the ultrasound. The limits were obtained by taking into account three plans of the space: transverse, vertical and anteroposterior. The sensibility of the ultrasound (capacity for this examination to identify the anatomical structures) was calculated. We also appreciated the axis of the gall-bladder.

The data were seized and analyzed on the software Ear information (version 6). The test of $X^{2}$ and Student were used to compare our results to those of the other authors. The threshold of significance was fixed to 0.05 .

\section{Results}

The average age was of $39 \pm 12.16$ with extreme of 10 years and 89 years. The 
distribution of the subjects by age bracket is represented by Figure 1. It was 500 women (50\%) and of 500 men (50\%) with a sex ratio of 1 .

700 sick ((70\%) lived in Bamako whereas 300 (30\%) came 8 regions of Mali.

The measurements made by the three sonographers showed no significant difference. The sensitivity of ultrasound in the visualization of the gallbladder was $100 \%$.

\subsection{The Length of the Gallbladder}

The length of the gall-bladder was between 61 and $70 \mathrm{~mm}$. The average was $65.35 \pm 14.48$. Extremes were 26 and $142 \mathrm{~mm}$.

Table 1 represents the average length of the gall-bladder of the subjects according to age groups.

There was not statistically significant difference for the average length of the subjects of the less of 20 years and the subjects of no more than 20 years $(p=0.071)$.

\subsection{The Width of the Gall-Bladder}

To 450 subjects (45\%) the width of the gall-bladder of the subjects varied between 21 and 30. The average was $22.63 \mathrm{~mm} \pm 7.9$. Extremes were from 9 to $55 \mathrm{~mm}$.

\subsection{The Thickness of the Gall-Bladder and Its Capacity}

Figure 2 represents the thickness of the wall of the vesicle and Figure 3 its capacity.

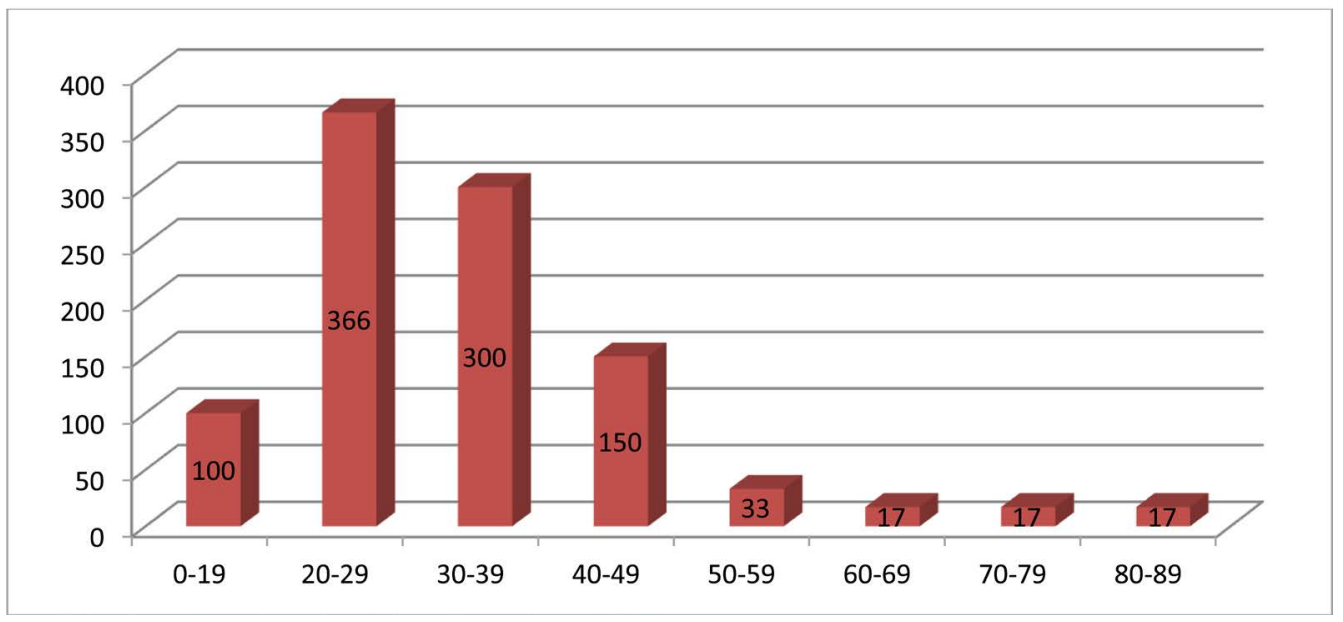

Figure 1. Distribution of subjects by age group.

Table 1. Length averages of the gall-bladder compared between the subjects of 20 years and the subjects of more than 20 years.

\begin{tabular}{cccc}
\hline Age (year) Dimensions $(\mathrm{mm})$ & $\leq 20(\mathrm{n}=133)$ & $>20(\mathrm{n}=867)$ & P value \\
\hline Length & $56.75 \pm 13.87 \mathrm{~mm}$ & $66.51 \pm 14.06 \mathrm{~mm}$ & 0.071 \\
Width & $21.62 \pm 6.42 \mathrm{~mm}$ & $22.54 \pm 8.10 \mathrm{~mm}$ & 0.760 \\
Wall thickness & $2.62 \pm 1.31 \mathrm{~mm}$ & $2.34 \pm 0.67 \mathrm{~mm}$ & 0.466 \\
\hline
\end{tabular}




\subsection{The Axis of the Gall-Bladder}

Table 2 and Figure 4 represent the axis of the gall-bladder.

\section{Discussion}

The dimensions of the gall-bladder were measured by three different sonographers in this protocol. We measured the dimensions of the gall-bladder (the length, the width, the thickness of the wall vesicular); the capacity of the gall-bladder was also measured. The limits of this study were the sampling was made according to a voluntarist mode. The second aspect is that if the gall-bladder is very cubit, its capacity can be modified by the fact of this coudure.

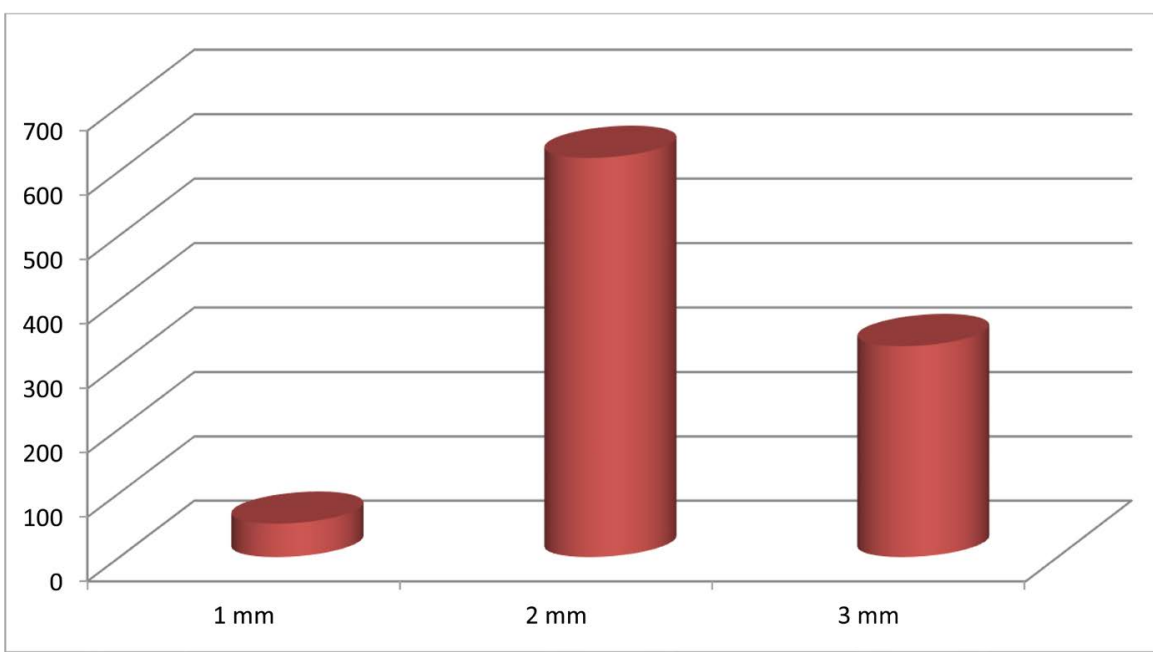

Figure 2. Thickness of the wall of the vesicle.

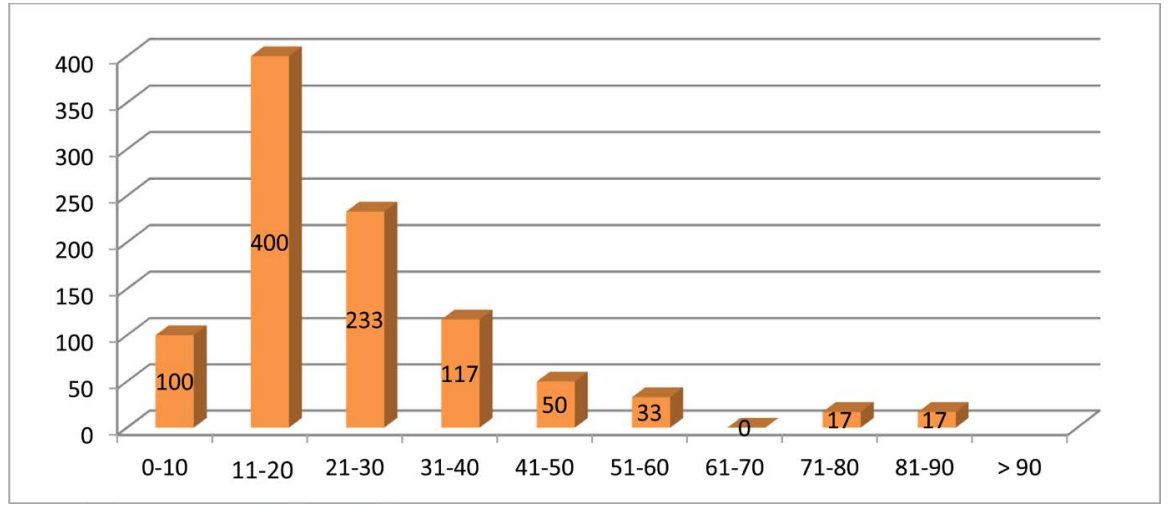

Figure 3. Patients according to the capacity of the gall-bladder.

Table 2. Distribution of the axis of the gall-bladder according to the sex.

\begin{tabular}{cccccc}
\hline \multirow{2}{*}{ Age } & \multicolumn{2}{c}{ Masculin } & \multicolumn{2}{c}{ Féminin } & Total \\
\cline { 2 - 5 } & Nombre & $\%$ & Nombre & $\%$ & \\
\hline Rectiligne & 341 & 53.9 & 292 & 46.1 & 633 \\
Coude & 159 & 43.3 & 208 & 56.7 & 367 \\
Total & 500 & & 500 & & 1000 \\
\hline
\end{tabular}




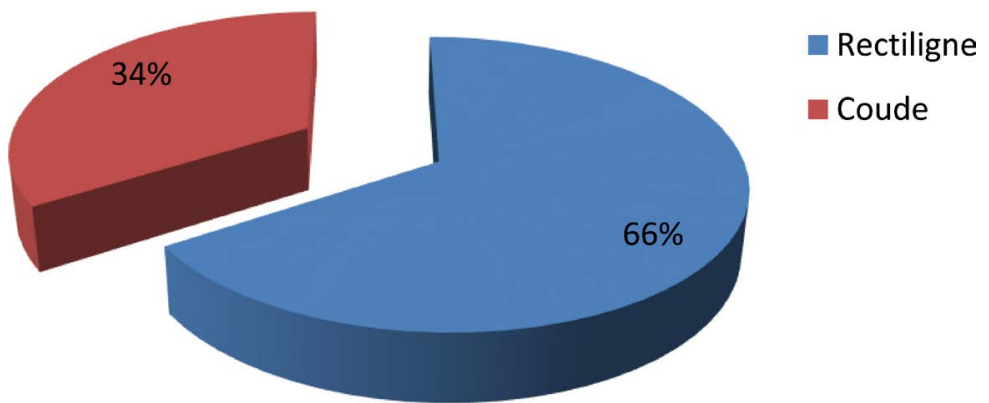

Figure 4. Aspect of the axis of the gall-bladder.

The dimensions and the capacity vesicular can be appreciated as well after anatomical dissection as by ultrasound. The gall-bladder is often approached by celio-surgery on the developed countries. It is often studied by ultrasound while its anatomical variations are not described at the African's yet. Indeed we did not find anatomical study realized about the gall-bladder in Africa.

The originality of our study is that we measured several variables at the same time and our sample is clearly upper to the standards usually used in the classic anatomical descriptions. Sometimes certain authors do not specify the number of subjects used for the anatomical descriptions [4] [5] [6]. So our anatomical study was realized on alive subjects contrary to the classic descriptions which are made on corpses sometimes kept for a long time. The results which we obtained are closer to the reality and allow giving to the surgeons a more exact orientation in the interventions.

The method of work was the same for all the subjects. Three sonographers used the same device and in the same conditions. The subjects were on an empty stomach 12 hours previously because the secretions of the present bile are a function of meals and the quantity of bile present in the gall-bladder can strongly influence the capacity of this one at the time of the ultrasound. The devices of ultrasound are not identical. Some are more precise than others. Marks devices are not always specified by the authors. We can say that the mark of the device which we used for this study was recent in the time. The gall-bladder for our three sonographers was easy to identify under the liver.

\section{Conclusion}

This original study shows the normal dimensions of the gall-bladder to Malian. These measures can help practitioners in the diagnosis of gallbladder pathologies.

\section{Authorization of the Ethics Committee}

The Ethics Committee of the Faculty of Medicine and Odontostomatologie gave us its agreement for the realization of this study.

The subjects on which were realized various measures gave their consent for the publication. 


\section{Conflicts of Interest}

We, authors of this article declare that there is no conflict of interests.

\section{References}

[1] Castaing, D. and Smail, A. (1999) Anatomy of the Liver and the Biliary Ways. Medical Surgical Encyclopedia, Hepatology, 7001, 12.

[2] Handler, S.J. (1972) Ultrasound of Gallbladder Wall Thickening and Its Relation Its Cholecystitis. American Journal of Radiology, 132, 581-585.

[3] Wenger, J.J., Foessel, M. and Warter, P. (1987) Conventional Radiological Exploration and Ultrasound of the Bilaterals. Medical-Surgical Encyclopedia. Paris Radiodiagnostic, 33500, 10.

[4] Delorme, G. and Gralet, P. (1975) Biliary Pathways. In Radiodiagnostic, Masson, Paris, No. 7, 25-151.

[5] Hiart, J.R., Gabay, J. and Busuth, R.W. (1994) Surgical Anatomy of the Hepatic Arteries in 1000 Cases. Annals of Surgery, 220, 50-52. https://doi.org/10.1097/00000658-199407000-00008

[6] Rouvière, H. (1985) Descriptive, Topographic and Functional Human Anatomy Reviewed and Augmented by A Delmas. 12th Edition, Paris, 696. 